\title{
Certain $p l(m, n)$-Kummer Matrix Function of Two Complex Variables under Differential Operator
}

\author{
Ayman Shehata ${ }^{1,2}$ \\ ${ }^{1}$ Department of Mathematics, Faculty of Science, Assiut University, Assiut, Egypt \\ ${ }^{2}$ Department of Basic Applied Sciences, Unaizah Community College, Qassim University, \\ Qassim, KSA \\ Email: drshehata2006@yahoo.com
}

Received October 3, 2012; revised November 3, 2012; accepted November 11, 2012

\begin{abstract}
The main aim of this paper is to define and study of a new matrix functions, say, the $p l(m, n)$-Kummer matrix function of two complex variables. The radius of regularity, recurrence relation and several new results on this function are established when the positive integers $p$ is greater than one. Finally, we obtain a higher order partial differential equation satisfied by the $\operatorname{pl}(m, n)$-Kummer matrix function and some special properties.
\end{abstract}

Keywords: Hypergeometric Matrix Function; $p l(m, n)$-Kummer Matrix Function; Matrix Differential Equation; Differential Operator

\section{Introduction}

Many Special matrix functions appear in connection with statistics [1], mathematical physics, theoretical physics, group representation theory, Lie groups theory [2] and orthogonal matrix polynomials are closely related [3-5]. The hypergeometric matrix function has been introduced as a matrix power series and an integral representation and the hypergeometric matrix differential equation in [6-9] and the explicit closed form general solution of it has been given in [10]. The author has earlier studied the Kummer's and Horn's $\mathrm{H}_{2}$ matrix function of two complex variables under differential operators [11-13]. In [14-16], extension to the matrix function framework of the classical families of $p$-Kummer's matrix function, $p$ and $q$-Appell matrix function and Humbert matrix function have been proposed.

Throughout this paper for a matrix $A$ in $C^{N \times N}$, its spectrum $\sigma(A)$ denotes the set of all the eigenvalues of $A$. If $A$ is a matrix in $C^{N \times N}$, its two-norm denoted by $\|A\|_{2}$ is defined by [17]

$$
\|A\|_{2}=\sup _{x \neq 0} \frac{\|A x\|_{2}}{\|x\|_{2}}
$$

where for a vector $y$ in $C^{N},\|y\|_{2}=\left(y^{\mathrm{T}} y\right)^{\frac{1}{2}}$ is the Euclidean norm of $y$.

If $f(z)$ and $g(z)$ are holomorphic functions of the complex variable $z$, defined in an open set $\Omega$ of the complex plane, and if $A$ and $B$ are a matrix in
$C^{N \times N}$ with $\sigma(A) \subset \Omega$ and $\sigma(B) \subset \Omega$ also and if $A B=B A$, then from the properties of the matrix functional calculus [18], it follows that

$$
f(A) g(B)=g(B) f(A) .
$$

The reciprocal gamma function denoted by $\Gamma^{-1}(z)=\frac{1}{\Gamma(z)}$ is an entire function of the complex variable $z$. Then for any matrix $A$ in $C^{N \times N}$, the image of $\Gamma^{-1}(z)$ acting on $A$ denoted by $\Gamma^{-1}(A)$ is a well defined matrix. Furthermore, if

$$
\begin{aligned}
& A+n I \text { is invertible for every } \\
& \text { non-negative integer } n
\end{aligned}
$$

where $I$ is the identity matrix in $C^{N \times N}$, then $\Gamma(A)$ is invertible, its inverse coincides with $\Gamma^{-1}(A)$ and one gets [6]

$$
\begin{aligned}
(A)_{n} & =A(A+I)(A+2 I) \cdots(A+(n-1) I) \\
& =\Gamma(A+n I) \Gamma^{-1}(A) ; n \geq 1 ;(A)_{0}=I .
\end{aligned}
$$

Jódar and Cortés have proved in [6], that

$$
\Gamma(A)=\lim _{n \rightarrow \infty}(n-1) !\left[(A)_{n}\right]^{-1} n^{A} .
$$

\section{On $p l(m, n)$-Kummer Matrix Function}

We We define the $p l(m, n)$-Kummer matrix function ${ }^{P} \Phi_{2}(A ; B ; z, w)$ of two complex variables in the form 


$$
\begin{aligned}
& { }^{P} \Phi_{2}(A ; B ; z, w) \\
= & \sum_{l(m, n) \geq 0} \frac{(A)_{l(m, n)}\left[(B)_{l(m, n)}\right]^{-1}}{(p l(m, n)) !} z^{m} w^{n} \\
= & \sum_{l(m, n) \geq 0} U_{m, n}(z, w)
\end{aligned}
$$

where

$$
\begin{aligned}
& U_{m, n}(z, w)=V_{m, n} z^{m} w^{n}, \\
& V_{m, n}=\frac{(A)_{l(m, n)}\left[(B)_{l(m, n)}\right]^{-1}}{(p l(m, n)) !} \text {, } \\
& \frac{1}{R}=\lim _{m+n \rightarrow \infty} \sup \left(\frac{\left\|V_{m, n}\right\|}{\sigma_{m, n}}\right)^{\frac{1}{m+n}}=\lim _{m+n \rightarrow \infty} \sup \left(\frac{\left\|(A)_{l(m, n)}\left[(B)_{l(m, n)}\right]^{-1}\right\|}{(p l(m, n)) ! \sigma_{m, n}}\right)^{\frac{1}{m+n}} \\
& =\lim _{m+n \rightarrow \infty} \sup \left\|\sqrt{2 \pi(A+(l(m, n)-1) I)}\left(\frac{(A+(l(m, n)-1) I)}{e}\right)^{(A+(l(m, n)-1) I)}\right\|^{\frac{1}{m+n}} \\
& \left.\times \| \sqrt{2 \pi(B+(l(m, n)-1) I)}\left(\frac{(B+(l(m, n)-1) I)}{e}\right)^{(B+(l(m, n)-1) I)}\right]^{-1} \|^{\frac{1}{m+n}} \\
& \times\left\|\left[\sqrt{2 p \pi t(m, n)}\left(\frac{p l(m, n)}{e}\right)^{p l(m, n)}\right]^{-1} \frac{1}{\sigma_{m, n}}\right\|^{\frac{1}{m+n}} \\
& \leq \lim _{m+n \rightarrow \infty} \sup \left\|\left(\frac{(A+(l(m, n)-1) I)}{e}\right)^{(A-I)}\left(\frac{(A+(l(m, n)-1) I)}{e}\right)^{l(m, n) I}\right\| \frac{1}{m+n} \\
& \times\left\|\left(\frac{(B+(l(m, n)-1) I)}{e}\right)^{-B+I}\left(\frac{(B+(l(m, n)-1) I)}{e}\right)^{-l(m, n) I}\left(\frac{(p l(m, n))}{e}\right)^{-(p l(m, n))}\right\|^{\frac{1}{m+n}} \\
& \leq \lim _{m+n \rightarrow \infty} \sup \left\|\left(\frac{(A+(l(m, n)-1) I)}{l(m, n)}\right)^{l(m, n) I}\right\|^{\frac{1}{m+n}} \times\left\|\left(\frac{(B+(l(m, n)-1) I)}{l(m, n)}\right)^{-l(m, n) I}\left(\frac{e}{p l(m, n)}\right)^{p l(m, n)}\right\|^{\frac{1}{m+n}} \\
& \leq \lim _{m+n \rightarrow \infty} \sup \left\|\left(I+\frac{A-I}{l(m, n)}\right)^{l(m, n) I}\right\| \frac{1}{m+n} \times\left(I+\frac{B-I}{l(m, n)}\right)^{-l(m, n) I}\left(\frac{e}{p l(m, n)}\right)^{p l(m, n)} \|^{\frac{1}{m+n}}=0 .
\end{aligned}
$$


where

$$
\sigma_{m, n}= \begin{cases}\left(\frac{m+n}{m}\right)^{\frac{m}{2}}\left(\frac{m+n}{n}\right)^{\frac{n}{2}}, & m, n \neq 0 ; \\ 1, & m, n=0 .\end{cases}
$$

Summarizing, the following result has been established.

Theorem 2.1. Let $A$ and $B$ be matrices in $C^{N \times N}$ such that $B+l(m, n) I$ are invertible for all integer $l(m, n) \geq 0$. Then, the $p l(m, n)$-Kummer matrix function is an entire function.

For $p=1$, we have

$$
\begin{aligned}
\frac{1}{R} & =\lim _{m+n \rightarrow \infty} \sup \left(\frac{\left.\left\|V_{m, n}\right\|\right)^{\frac{1}{\sigma_{m, n}}}}{{ }^{m+n}}\left(\left\|(A)_{l(m, n)}\left[(B)_{l(m, n)}\right]^{-1}\right\|\right)^{\frac{1}{m+n}}=0\right. \\
& =\lim _{m+n \rightarrow \infty} \sup \left(\frac{\|(m, n) ! \sigma_{m, n}}{{ }^{m}}=0\right.
\end{aligned}
$$

i.e., the $l(m, n)$-Kummer matrix function is an entire function.

Some matrix recurrence relations are carried out on the $p l(m, n)$-Kummer matrix function. In this connection the following matrix contiguous functions relations follow, directly by increasing or decreasing one in original relation

$$
\begin{aligned}
& { }^{p} \Phi_{2}(A+; B ; z, w) \\
& =\sum_{l(m, n) \geq 0} \frac{(A+I)_{l(m, n)}\left[(B)_{l(m, n)}\right]^{-1}}{(p l(m, n)) !} z^{m} w^{n} \\
& =\sum_{l(m, n) \geq 0} \frac{A^{-1}(A+l(m, n) I)(A)_{l(m, n)}\left[(B)_{l(m, n)}\right]^{-1}}{(p l(m, n)) !} z^{m} w^{n} \\
& =A^{-1} \sum_{l(m, n) \geq 0}(A+l(m, n) I) U_{m, n}(z, w) . \\
& { }^{p} \Phi_{2}(A-; B ; z, w) \\
& =\sum_{l(m, n) \geq 0}(A-I)[(A+(l(m, n)-1) I)]^{-1} U_{m, n}(z, w), \\
& { }^{p} \Phi_{2}(A ; B+; z, w) \\
& =\sum_{l(m, n) \geq 0} B[(B+l(m, n) I)]^{-1} U_{m, n}(z, w), \\
& { }^{p} \Phi_{2}(A ; B-; z, w) \\
& =\sum_{l(m, n) \geq 0}(B-I)^{-1}(B+(l(m, n)-1) I) U_{m, n}(z, w) .
\end{aligned}
$$

By the same way, we have

$$
\begin{aligned}
& { }^{p} \Phi_{2}(A+; B+)=A^{-1} B \sum_{l(m, n) \geq 0}(A+l(m, n) I)[(B+l(m, n) I)]^{-1} U_{m, n}(z, w), \\
& { }^{p} \Phi_{2}(A+; B-)=A^{-1}(B-I)^{-1} \sum_{l(m, n) \geq 0}(A+l(m, n) I)(B+(l(m, n)-1) I) U_{m, n}(z, w), \\
& { }^{p} \Phi_{2}(A-; B+)=B(A-I) \sum_{l(m, n) \geq 0}[(A+(l(m, n)-1) I)]^{-1}[(B+l(m, n) I)]^{-1} U_{m, n}(z, w), \\
& { }^{p} \Phi_{2}(A-; B-)=(A-I)(B-I)^{-1} \sum_{l(m, n) \geq 0}[(A+(l(m, n)-1) I)]^{-1}(B+(l(m, n)-1) I) U_{m, n}(z, w) .
\end{aligned}
$$

Now, we consider the following differential operators

$$
D=\frac{1}{2}(D)_{2}+d_{2}=\frac{1}{2}\left(D^{2}+D\right)+d_{2}
$$

where $D=d_{1}+d_{2}, d_{1}=z \frac{\partial}{\partial z}$ and $d_{2}=w \frac{\partial}{\partial w}$.

It is clear that

$$
\begin{aligned}
& D^{p} \Phi_{2}(A ; B ; z, w)=\left[\frac{1}{2}\left(D^{2}+D\right)+d_{2}\right]{ }^{p} \Phi_{2}(A ; B ; z, w)=\sum_{l(m, n) \geq 0} \frac{\left[\frac{1}{2}\left((m+n)^{2}+(m+n)\right)+n\right](A)_{l(m, n)}\left[(B)_{l(m, n)}\right]^{-1}}{(p l(m, n)) !} z^{m} w^{n} \\
& =\sum_{l(m, n) \geq 0} \frac{\left[\frac{1}{2}((m+n)(m+n+1))+n\right](A)_{l(m, n)}\left[(B)_{l(m, n)}\right]^{-1}}{(p l(m, n)) !} z^{m} w^{n}=\sum_{l(m, n) \geq 0} \frac{l(m, n)(A)_{l(m, n)}\left[(B)_{l(m, n)}\right]^{-1}}{(p l(m, n)) !} z^{m} w^{n} .
\end{aligned}
$$


So that

$$
\begin{aligned}
& D^{p} \Phi_{2}(A ; B ; z, w) \\
& =\sum_{l(m, n) \geq 0} \frac{l(m, n)(A)_{l(m, n)}\left[(B)_{l(m, n)}\right]^{-1}}{(p l(m, n)) !} z^{m} w^{n} \\
& =\frac{1}{p} \sum_{l(m, n) \geq 0} \frac{(A)_{l(m, n)}\left[(B)_{l(m, n)}\right]^{-1}}{[(p l(m, n))-1] !} z^{m} w^{n} .
\end{aligned}
$$

Putting in this relation $m-1$ and $n+1$ instead of $m$ and $n$ respectively, then

$$
l(m-1, n+1)=\frac{1}{2}(m+n)(m+n+1)+n+1=l(m, n)+1
$$

and so that we can be written the relation $m-\frac{1}{p}$ and $n+\frac{1}{p}$ instead of $m$ and $n$ yields

$$
\begin{aligned}
& l\left(m-\frac{1}{p}, n+\frac{1}{p}\right)=l(m, n)+\frac{1}{p} \\
& \text { and } p l\left(m-\frac{1}{p}, n+\frac{1}{p}\right)=p l(m, n)+1 .
\end{aligned}
$$

Therefore, the power series ${ }^{p} \Phi_{2}(A ; B ; z, w)$, as follows

$$
\begin{aligned}
& {\left[D\left(D-\frac{1}{p}\right)\left(D-\frac{2}{p}\right) \cdots\left(D-\frac{p-1}{p}\right)\right]{ }^{p} \Phi_{2}(A ; B ; z, w)} \\
& =\sum_{l(m, n) \geq 0} \frac{l(m, n)\left[l(m, n)-\frac{1}{p}\right]\left[l(m, n)-\frac{2}{p}\right] \ldots\left[l(m, n)-\frac{p-1}{p}\right](A)_{l(m, n)}\left[(B)_{l(m, n)}\right]^{-1}}{(p l(m, n)) !} z^{m} w^{n} \\
& =\frac{1}{p^{p}} \sum_{l l(m, n) \geq 0} \frac{(A)_{l(m, n)}\left[(B)_{l(m, n)}\right]^{-1}}{(p l(m, n)-p) !} z^{m} w^{n}=\frac{1}{p^{p}} \sum_{l l(m, n) \geq 0} \frac{(A)_{l(m, n)+1}\left[(B)_{l(m, n)+1}\right]^{-1}}{(p l(m, n)) !} z^{m-1} w^{n+1} \\
& =\frac{w}{z} \frac{1}{p^{p}} \sum_{l(m, n) \geq 0} \frac{(A)_{l(m, n)+1}\left[(B)_{l(m, n)+1}\right]^{-1}}{(p l(m, n)) !} z^{m} w^{n}=\frac{w}{z} \frac{1}{p^{p}} \sum_{l(m, n) \geq 0} \frac{[A+l(m, n) I][B+l(m, n) I]^{-1}(A)_{l(m, n)}\left[(B)_{l(m, n)}\right]^{-1}}{(p l(m, n)) !} z^{m} w^{n} \\
& =\frac{w}{z} \frac{1}{p^{p}} \sum_{l(m, n) \geq 0}[A+l(m, n) I][B+l(m, n) I]^{-1} \frac{(A)_{l(m, n)}\left[(B)_{l(m, n)}\right]^{-1}}{(p l(m, n)) !} z^{m} w^{n} \\
& =\frac{w}{z} \frac{1}{p^{p}} \sum_{l(m, n) \geq 0} \frac{(A)_{l(m, n)}\left[(B)_{l(m, n)}\right]^{-1}}{(p l(m, n)) !} z^{m} w^{n} \\
& +\frac{w}{z} \frac{1}{p^{p}} \sum_{l(m, n) \geq 0}(A-B)[B+l(m, n) I]^{-1} \frac{(A)_{l(m, n)}\left[(B)_{l(m, n)}\right]^{-1}}{(p l(m, n)) !} z^{m} w^{n} \\
& =\frac{w}{z} \frac{1}{p^{p}}{ }^{p} \Phi_{2}(A ; B ; z, w)+\frac{w}{z} \frac{1}{p^{p}}(A-B) B^{-1}{ }^{p} \Phi_{2}(A ; B+; z, w)
\end{aligned}
$$

i.e., the $p l(m, n)$-Kummer matrix function is a solution of the matrix differential equation

$$
\begin{aligned}
& D^{p} \Phi_{2}(A ; B ; z, w) \\
& -\frac{1}{p}\left(\frac{w}{z}\right)^{\frac{1}{p}}(A)[(B)]^{-1} \Phi_{2}\left(A+\frac{1}{p} I ; B+\frac{1}{p} I ; z, w\right)=0 .
\end{aligned}
$$

In this paper, we affect by differential operator $D$ the $p l(m, n)$-Kummer matrix function, successively, then we have 
i.e. the $(m, n)$-Kummer matrix function is a solution to this matrix differential equation

$$
\left[D\left(D-\frac{1}{p}\right)\left(D-\frac{2}{p}\right) \cdots\left(D-\frac{p-1}{p}\right)-\frac{w}{z} \frac{1}{p^{p}}\right]{ }^{p} \Phi_{2}(A ; B ; z, w)-\frac{w}{z} \frac{1}{p^{p}}(A-B){ }^{p} \Phi_{2}(A ; B+; z, w)=0 .
$$

Then

$$
\begin{aligned}
& {\left[D\left(D-\frac{1}{p}\right)\left(D-\frac{2}{p}\right) \ldots\left(D-\frac{p-1}{p}\right)(D I+B-I)\right]{ }^{p} \Phi_{2}(A ; B ; z, w)} \\
& =\sum_{l(m, n) \geq 0} \frac{l(m, n)\left[l(m, n)-\frac{1}{p}\right]\left[l(m, n)-\frac{2}{p}\right] \ldots\left[l(m, n)-\frac{p-1}{p}\right](B+(l(m, n)-1) I)(A)_{l(m, n)}\left[(B)_{l(m, n)}\right]^{-1}}{(p l(m, n)) !} z^{m} w^{n} \\
& =\frac{1}{p^{p}} \sum_{l(m, n) \geq 0} \frac{(B+(l(m, n)-1) I)(A)_{l(m, n)}\left[(B)_{l(m, n)}\right]^{-1}}{(p l(m, n)-p) !} z^{m} w^{n}=\frac{1}{p^{p}} \sum_{l(m, n) \geq 0} \frac{(B+l(m, n) I)(A)_{l(m, n)+1}\left[(B)_{l(m, n)+1}\right]^{-1}}{(p l(m, n)) !} z^{m-1} w^{n+1} \\
& =\frac{w}{z} \frac{1}{p^{p}} \sum_{l(m, n) \geq 0} \frac{(B+l(m, n) I)(A)_{l(m, n)+1}\left[(B)_{l(m, n)+1}\right]^{-1}}{(p l(m, n)) !} z^{m} w^{n}=\frac{w}{z} \frac{1}{p^{p}} \sum_{l(m, n) \geq 0} \frac{[A+l(m, n) I](A)_{l(m, n)}\left[(B)_{l(m, n)}\right]^{-1}}{(p l(m, n)) !} z^{m} w^{n} \\
& =\frac{w}{z} \frac{1}{p^{p}} A^{p} \Phi_{2}(A ; B ; z, w) .
\end{aligned}
$$

Therefore, the following result has been established.

Theorem 2.2. Let $A$ and $B$ be matrices in $C^{N \times N}$. Then the $p l(m, n)$-Kummer matrix function is solution of this matrix differential equation

$$
\begin{aligned}
& {\left[D\left(D-\frac{1}{p}\right)\left(D-\frac{2}{p}\right) \cdots\left(D-\frac{p-1}{p}\right)(D I+B-I)\right.} \\
& \left.-\frac{w}{z} \frac{1}{p^{p}} A\right]{ }^{p} \Phi_{2}(A ; B ; z, w)=0 .
\end{aligned}
$$

The $\alpha(D)$ differential operator has been defined by Sayyed [19] in the form

$$
\alpha(D)=1+\sum_{k=1}^{N} D^{k} ; D^{k}=D D^{k-1} .
$$

From (2.1), (2.3) and (2.5), we obtain

$$
\begin{aligned}
& (D I+B-I)^{p} \Phi_{2}(A ; B ; z, w) \\
& =\sum_{l(m, n) \geq 0} \frac{(B+(l(m, n)-1) I)(A)_{l(m, n)}\left[(B)_{l(m, n)}\right]^{-1}}{(p l(m, n)) !} z^{m} w^{n} \\
& =\sum_{l(m, n) \geq 0} \frac{(B-I)(A)_{l(m, n)}\left[(B-I)_{l(m, n)}\right]^{-1}}{(p l(m, n)) !} z^{m} w^{n} \\
& =(B-I)^{p} \Phi_{2}(A ; B-I ; z, w)
\end{aligned}
$$

hence

$$
\begin{aligned}
& D^{p} \Phi_{2}(A ; B ; z, w) \\
& =(B-I)\left[{ }^{p} \Phi_{2}(A ; B-I ; z, w)-{ }^{p} \Phi_{1}(A ; B ; z, w)\right]
\end{aligned}
$$

and

$$
\begin{aligned}
& D^{2}{ }^{p} \Phi_{2}(A ; B ; z, w) \\
& =(B-I)(B-2 I)^{p} \Phi_{2}(A ; B-2 I ; z, w) \\
& -\left[(B-I)(B-2 I)+(B-I)^{2}\right]^{p} \Phi_{2}(A ; B-I ; z, w) \\
& +(B-I)^{2}{ }^{p} \Phi_{2}(A ; B ; z, w) .
\end{aligned}
$$

Thus by mathematical induction, we have the following general form

$$
\begin{aligned}
& \alpha(D){ }^{p} \Phi_{2}(A ; B ; z, w)=\left(1+\sum_{k=1}^{N} D^{k}\right){ }^{p} \Phi_{2}(A ; B ; z, w) \\
& ={ }^{p} \Phi_{2}(A ; B ; z, w)+\sum_{k=1}^{N} \prod_{j=1}^{k}(B-j I)^{p} \Phi_{2}(A ; B-j I ; z, w) \\
& -\left[\prod_{j=1}^{k}(B-j I)+\prod_{j=1}^{k-1}(B-j I) \sum_{k=1}^{N-1}(B-j I)\right] \\
& +\left[\prod_{j=1}^{k-1}(B-j I) \sum_{j=1}^{k-1}(B-j I)+\prod_{j=1}^{k-2}(B-j I)\left(\sum_{j=1}^{k-2}(B-j I)\right)^{2}\right. \\
& +\sum_{j=1}^{k-3}(B-j I)(B-(j+1) I) \\
& \left.+\sum_{j=1}^{k-4}(B-j I)(B-(j+1) I)+\cdots\right] \\
& { }^{p} \Phi_{2}(A ; B-(j-2) I ; z, w)+\cdots \\
& +(-1)^{k}(B-I)^{k}{ }^{p} \Phi_{2}(A ; B ; z, w) .
\end{aligned}
$$


where $N$ is a finite positive integer.

Special cases: we can be written the matrix function $\Phi_{2}(A ;-; z, w)$ in the form

$$
{ }^{p} \Phi_{2}(A ;-; z, w)=\sum_{l l(m, n) \geq 0} \frac{(A)_{l(m, n)}}{(p l(m, n)) !} z^{m} w^{n}
$$

we see that

$$
D^{p} \Phi_{2}(A ;-; z, w)=\frac{w}{z}(D I+A){ }^{p} \Phi_{2}(A ;-; z, w) .
$$

i.e., the ${ }^{p} \Phi_{2}(A ;-; z, w)$ is a solution to this matrix differential equation

$$
\left[D\left(1-\frac{w}{z}\right) I-\frac{w}{z} A\right]{ }^{p} \Phi_{2}(A ;-; z, w)=0 .
$$

Also

$$
\begin{aligned}
& D^{p} \Phi_{2}(A ;-; z, w) \\
& =\frac{w}{z}(D I+A)^{p} \Phi_{2}(A ;-; z, w) \\
& +\frac{w^{2}}{z^{2}}(D I+A)(D I+A+I){ }^{p} \Phi_{2}(A ;-; z, w) .
\end{aligned}
$$

i.e., the ${ }^{p} \Phi_{2}(A ;-; z, w)$ is a solution for the matrix partial differential equations

$$
\begin{aligned}
& {\left[D^{2} I-\frac{w}{z}(D I+A)-\frac{w^{2}}{z^{2}}(D I+A)(D I+A+I)\right]} \\
& { }^{p} \Phi_{2}(A ;-; z, w)=0 .
\end{aligned}
$$

The results of this paper are variant, significant and so it is interesting and capable to develop its study in the future. One can use the same class of differential operators for some other function of several complex variables. Hence, new results and further applications can be obtained.

\section{Acknowledgements}

The Author expresses his sincere appreciation to Dr. M. S. Metwally, (Department of Mathematics, Faculty of Science (Suez), Suez Canal University, Egypt) for his kind interest, encouragements, help, suggestions, comments and the investigations for this series of papers.

The author would like to thank the referees for his comments and suggestions on the manuscript.

\section{REFERENCES}

[1] A. G. Constantine and R. J. Mairhead, "Partial Differential Equations for Hyper-Geometric Functions of Two Argument Matrices," Journal of Multivariate Analysis, Vol. 2, No. 3, 1972, pp. 332-338. doi:10.1016/0047-259X(72)90020-6

[2] A. T. James, "Special Functions of Matrix and Single Argument in Statistics in Theory and Application of Special Functions," Academic Press, New York, 1975.

[3] L. Jódar and J. C. Cortés, "Some Properties of Gamma and Beta Matrix Functions," Applied Mathematics Letters, Vol. 11, No. 1, 1998, pp. 89-93. doi:10.1016/S0893-9659(97)00139-0

[4] A. M. Mathai, "A Handbook of Generalized Special Functions for Statistical and Physical Sciences," Oxford University Press, Oxford, 1993.

[5] A. M. Mathai, "Jacobians of Matrix Transformations and Functions of Matrix Argument," World Scientific Publishing, New York, 1997.

[6] L. Jódar and J. C. Cortés, "On the Hypergeometric Matrix Function," Journal of Computational and Applied Mathematics, Vol. 99, No. 1-2, 1998, pp. 205-217. doi:10.1016/S0377-0427(98)00158-7

[7] K. A. M. Sayyed, M. S. Metwally and M. T. Mohamed, "Certain Hypergeometric Matrix Function," Scientiae Mathematicae Japonicae, Vol. 69, No. 3, 2009, pp. 315-321. http://www.jams.or.jp/notice/scmjol/2009.html\#2009-21

[8] M. T. Mohamed and A. Shehata, "A Study of Appell's Matrix Functions of Two Complex Variables and Some Properties," Advances and Applications in Mathematical Sciences, Vol. 9, No. 1, 2011, pp. 23-33.

[9] Z. M. G. Kishka, A. Shehata and M. Abul-Dahab, "A New Extension of Hypergeometric Matrix Functions," Advances and Applications in Mathematical Sciences, Vol. 10, No. 4, 2011, pp. 349-371.

[10] L. Jódar and J. C. Cortés, "Closed form General Solution of the Hypergeometric Matrix Differential Equation," Mathematical and Computer Modelling, Vol. 32, No. 9, 2000, pp. 1017-1028. doi:10.1016/S0895-7177(00)00187-4

[11] A. Shehata, "A Study of Some Special Functions and Polynomials of Complex Variables," Ph.D. Thesis, Assiut University, Assiut, 2009.

[12] A. Shehata, "On $p$ - and $q$-Horn's Matrix Function of Two Complex Variables," Applied Mathematics, Vol. 2, No. 12, 2011, pp. 1437-1442. doi:10.4236/am.2011.212203

[13] A. Shehata, "On Pseudo Legendre Matrix Polynomials," International Journal of Mathematical Sciences and Engineering Applications (IJMSEA), Vol. 6, No. 6, 2012, pp. 251-258.

[14] Z. M. G. Kishka, M. A. Saleem, S. Z. Radi and M. AbulDahab, "On the $p$ - and $q$-Appell Matrix Function," SouthEast Asian Bulletin of Mathematics, Vol. 35, 2011, pp. 807-818.

[15] M. S. Metwally, "On $p$-Kummers Matrix Function of Complex Variable under Differential Operators and Their Properties," South-East Asian Bulletin of Mathematics, Vol. 35, 2011, pp. 1-16.

[16] A. Shehata and M. Abul-Dahab, "A New Extension of Humbert Matrix Function and Their Properties," $A d$ vances in Pure Mathematics, Vol. 1, No. 6, 2011, pp. 315321. doi:10.4236/apm.2011.16057

[17] G. Golub and C. F. Van Loan, "Matrix Computations," The Johns Hopkins University Press, Baltimore, 1989.

[18] N. Dunford and J. Schwartz, "Linear Operators, Part I," Interscience, New York, 1955.

[19] K. A. M. Sayyed, "Basic Sets of Polynomials of Two Complex Variables and Convergence Properties," Ph.D. Thesis, Assiut University, Assiut, 1975. 